DOE Document Number DOE/ER/13958-30

SEMIEMPIRICAL STUDIES OF ATOMIC STRUCTURE

U.S. Department of Energy, Fundamental Interactions Brancn Division of Chemical Sciences, Office of Basic Energy Sciences

Grant Number DE-FG05-88ER13958

\author{
L. J. Curtis \\ Department of Physics and Astronomy \\ University of Toledo \\ 2801 W. Bancroft Street \\ Toledo, OH 43606 \\ tel. (419) 537-2341 \\ fax (419) 537-2723 \\ bitnet fac0283 @ uoft01 \\ email: ljc @ utphya.phya.utoledo.edu
}

\title{
DISCLAIMER
}

This report was prepared as an account of work sponsored by an agency of the United States Government. Neither the United States Government nor any agency thereof, nor any of their employees, makes any warranty, express or implied, or assumes any legal liability or responsibility for the accuracy, completeness, or usefulness of any information, apparatus, product, or process disclosed, or represents that its use would not infringe privately owned rights. Reference herein to any specific commercial product, process, or service by trade name, trademark, manufacturer, or otherwise does not necessarily constitute or imply its endorsement, recommendation, or favoring by the United States Government or any agency thereof. The views and opinions of authors expressed herein do not necessarily state or reflect those of the United States Government or any agency thereof. 


\section{SCOPE OF INVESTIGATIONS}

The energy level structure, transition probabilities, and general spectro scopic properties of highly-ionized many-electron systems are studied through the combined use of sensitive semiempirical data systematizations, selected precision experimental measurements, and specia!ized theoretical computations. Measurements are made primarily through the use of fast ion beam excitation methods, which are combined with available data from laser and tokamak produced plasmas, astrophysical sources, and conventional light sources. The experimental studies are strengthened through large scale $a b$ initio calculations. Large blocks of data are predictively systematized and parametrized along isoelectronic, homologous, isoionic, Rydberg, and yrast series, to provide a comprehensive and reliable data base.

\section{SIGNIFICANT RESULTS}

During the period from 1 March 1992 - 15 December 1992, work done as part of this project has resulted in eleven papers published or submitted for publication [1-11]. This has been done using experimental fast-ion-beam measurements, theoretical calculations, and semiempirical systematizations of spectroscopic data. A few typical examples will be described below.

Lifetime measurements in the neon isoelectronic sequence. Because of the strong radiative decay of the $2 p^{6}-2 p^{5} 3 s$ transitions in the $\mathrm{Ne}$ isoelectronic sequence, it is possible to create a population inversion between the $2 \mathrm{p}^{5} 3 \mathrm{~s}$ and $2 p^{5} 3 p$ levels which can produce XUV laser amplification action. Modeling studies have demonstrated that anplification is optimal near $Z=36$, leading to applications to Se and Y plasmas. The lifetimes of these levels are too short for time-of-flight measurements at $Z=36$, but we have developed methods that permit reliable semiempirical extrapolation if precise results at lower $Z$ are available. We have performed precision measurements of the $2 p^{6}-2 p^{5} 3 s-$ $2 p^{5} 3 p$ transitions in S VII and Cl VIII. These studies were made possible by the existence of a uniquely designed beam-foil chamber at the University of Lund which views a very short segment of beam with a grazing incidence monochromator, permitting the precision measurement of lifetimes as short as few ps for wavelengths well below $100 \AA$. These measurements yielded lifetimes accurate to $3-4 \%$, as compared to earlier measurements which had uncertainties $\geq 25 \%$. In addition to the curve fits, it was also possible to perform ANDC correlated decay analyses with the primary cascade channel $2 p^{5} 3 s-2 p^{5} 3 p$ (the laser transition). We have applied a semiempirical technique which determines an effective singlet-triplet mixing angle $\vartheta$ from spectroscopic energy level data, and divides the line strength of the resonance line by $\cos ^{2} \vartheta$ and that of the intercombination line by $\sin ^{2} \vartheta$. In earlier studies of a variety of isoelectronic sequences, this plot has been found to be empirically linear, and if extrapolated to $\mathrm{Se}(Z=34)$ it implies lifetimes for the resonance and intercombination lines of 0.13 and 0.55 ps. While this approach is somewhat speculative, existing $a b$ initio calculations vary by factors of 2 , and a semiempirical approach that is extrapolatively reliable to the $3-4 \%$ levels of accuracy provided by our measurements is valuable for modeling purposes. 
Multiplexed decay curve measurements of Li-like Si XII. We have utilized position sensitive detection of VUV emission from excited fast silicon ions to make precision measurements of the $J$ dependence of the lifetimes of the $2 s-2 p$ multiplet in Li-like Si XII. The experiment was carried out at the Notre Dame Tandem Accelerator in collaboration with A.E. Livingston of Notre Dame and H.G. Berry of ANL. The $42 \mathrm{MeV}$ foil-excited Si beam was viewed with a $1 \mathrm{~m}$ normal incidence monochromator, using a detector consisting of a 1 inch wide windowless channeled electron multiplier array in the chevron configuration, coupled with a resistive anode position sensor. The position decoding of the photon induced pulses was obtained by comparison of the divided pulse charges appearing at the two ends of the anode. The two strongest lines are the $2 \mathrm{~s}-2 \mathrm{p}$ fine structure resonance transitions (in 2nd order), and the other features arise from the Si XII $n=5-6$ and $S i$ XI $n=6-7$ Rydberg transition complexes. This multiplexed registration provides a more precise determination of the relative lifetimes through the cancellation of the small systematic effects (beam fluctuations, degradation of foil quality, etc) that limit the accuracy of separately measured decay curves. When reduced to eliminate $\lambda^{3}$ dependences, the measured line strength ratio was $\mathrm{S}(1 / 2-3 / 2): \mathrm{S}(1 / 2-1 / 2)=1.979(44)$, which can be compared with ab initio calculations of 2.007 (MBPT) and 2.005 (MCDF), and a semiempirical calculation of 2.007 (CAHS). This type of multiplexed decay curve measurement offers new possibilities for improved accuracy in lifetime measurement. As one example, it could permit sensitive measurements of the relative lifetimes of fine structure components where CI or IC selectively couples a particular $J$ level to produce an additional intercombination or autoionization channel. It also makes possible the real-time application of the ANDC correlated analysis of cascade repopulation for a system if the primary and cascade transitions are within the same wavelength range.

Isoelectronic specification of intershell resonance and intercombination decay rates using measured transition probabilities and spectroscopically determined singlet-triplet mixing amplitudes. Methods recently developed to specify alkaliline-earthlike $\Delta n=0$ intrashell transitions of the form $\mathrm{ns}^{2}-\mathrm{nsnp}$ were extended to intershell transitions $2 s^{2}-2 s 3 p$ levels in the $B e$ isoelectronic sequence. These transitions provided a stringent test of these methods, since they are affected by plunging configuration interaction, transition integral cancellations, and multiple exit channels that were not present in the intrashell transitions. Experimental measurements of the intercombination transition probabilities were accessible by virtue differential decay curve measurements that exploit the $J$ dependence of the exit channels of the triplet levels. Using our own measurements for intercombination rates in $\mathrm{N} I \mathrm{~V}, 0 \mathrm{~V}$, $\mathrm{F} V I$, and Ne VII, a joint linearizing formulation was obtained that also predicts the lifetime of the resonance transition. The homologous counterpart given by the $3 s^{2}-3 s 4 p$ levels in the $M g$ sequence was also studied using existing measurements for $\mathrm{S} \mathrm{V}$ and $\mathrm{Cl}$ VI. Here the $3 p 3 d$ configuration introduced significant plunging configuration interaction and additional intercombination channels. This produces heavy CI effects in S V which are not present in $\mathrm{Cl}$ VI. It was possible to characterize the CI as indirect rather than direct because the singlet-triplet mixing amplitudes obtained from the spectroscopic data removed the isoelectronic irregularities from the reduced transition probability data. 


\section{BIBLIOGRAPHY OF PUBLICATIONS PRODUCED ON THIS PROJECT}

1 March 1992 - 15 December 1992

1. L.J. Curtis, "A Predictive Systematisation for $n s^{2}-n s n ' p$ line strength Data," J. Phys.B: Atomic, Molecular and Optical Physics 25, 1425-35 (1992). [Document DOE/ER/13958-28]

2. M. Westerlind, L. Engström, P. Bengtsson and L.J. Curtis, "Experimental Lifetimes of the $2 \mathrm{p}^{5} 3 \mathrm{~s}^{1} \mathrm{P}_{1}$ and ${ }^{3} \mathrm{P}_{1}$ Levels in Ne-like Sulfur and Chlorine," Phys. Rev. A 45, 6198-201 (1992). [Document DOE/ER/13958-29]

3. D.J. Beideck, L.J. Curtis, R.E. Irving, S.T. Maniak, R. Hellborg, S.G. Johansson, A. A. Joueizadeh, I. Martinson, and T. Brage, "Lifetimes of $5 d^{9} 6 p$ and $5 d^{8} 6 s 6 p$ Levels in Hg III," Phys. Rev. A (Accepted 27 October 1992).

4. S. Cheng, R.W. Dunford, E.P. Kanter, H.G. Berry, D.S. Gemmell, B. J. Zabransky, A.E. Livingston, L.J. Curtis, J. Baily, and J.A. Nolen, "Branching Ratio for the M1 Decay of the $2{ }^{2} \mathrm{~S} 1 / 2$ state in One-Electron Krypton," Phys. Rev. A (Submitted 31 August 1992).

5. R.W. Dunford, S. Cheng, E.P. Kanter, H.G. Berry, C. Kurtz, B. J. Zebransky, A.E. Livingston, and L.J. Curtis, "Two-Photon Decay of the $2{ }^{1}$ So State in Heliumlike Bromine," Phys. Rev. A (Submitted September 1992).

6. S.T. Maniak, E. Träbert, and L.J. Curtis, "Beam-Foil Lifetime Measurements of Low-Lying Levels in Si IV," Phys. Lett. A (Submitted 30 September 1992).

7. R.R. Haar and L.J. Curtis, "ACCEL, A Program for Data Acquisition," Nuclear Instruments and Methods in Physics Research B (Submitted 2 November 1992).

8. R.R. Haar, D.J. Beideck, L.J. Curtis, T.J. Kvale, A. Sen, R.M. Schectman, and $H$. Stevens, "The Toledo Heavy Ion Accelerator," Nuclear Instruments and Methods in Physics Research B. (Submitted 2 November 1992).

9. D.J. Beideck, L.J. Curtis, R.E. Irving, S.T. Maniak, R. Hellborg, S.G. Johansson, I. Martinson, and M. Rosberg, "Lifetimes of $5 d^{9} 6 p$ levels in Au II," Journal of the Optical Society of America (Submitted 1 November $1992)$.

10. E. Träbert, H.G. Berry, R.W. Dunford, J. Sulieman, E.W. Kanter, C. Kurtz, S. Cheng, A.E. Livingston, K.W. Kukla, F.G. Serpa, and L.J. Curtis, Experimental Transition Probabilities of Intercombination Transitions in Mg-like, Al-like, and Si-like Ions of Bromine," Phys. Rev. A (submitted November 1992).

11. K.W. Kukla, A.E. Livingston, F.G. Serpa, A.S. Zacarias, H.G. Berry, R.W. Dunford, E. Kanter, S. Cheng, J. Suleiman, L. J. Curtis, and E. Träbert, "Spectroscopy of Highly-Ionized Atoms Using Position-Sensitive Detection," AIP Conference Proceedings (in press). 

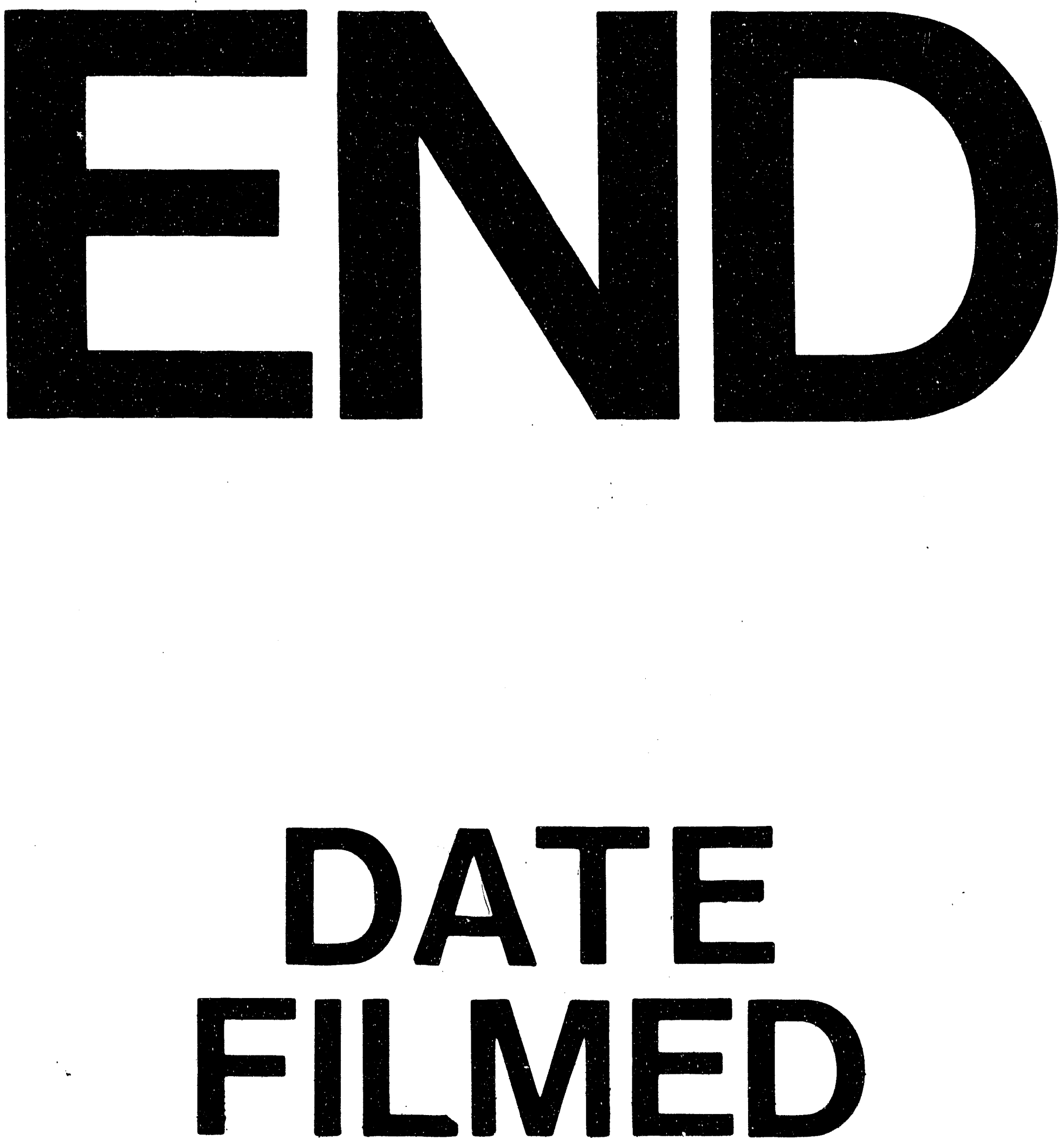

I

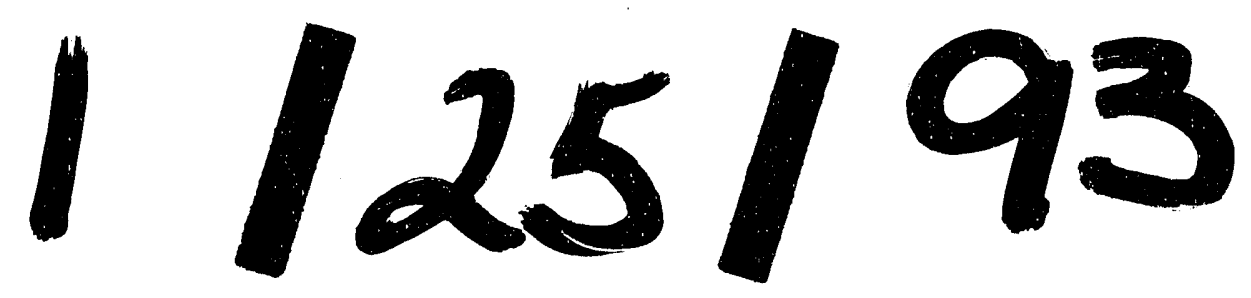


